\title{
Examining Prevalence of Cancer Risk Factors Across Ontario for the Ontario Cancer Profiles Tool
}

\author{
Amy Chang ${ }^{1}$, Naomi Schwartz ${ }^{2}$ and Rebecca Truscott ${ }^{2}$ \\ ${ }^{1}$ Dalla Lana School of Public Health, University of Toronto, Toronto, Canada \\ ${ }^{2}$ Ontario Health (Cancer Care Ontario), Toronto, Canada. \\ E-mail: amy.chang@mail.utoronto.ca
}

Received October 28, 2020

Accepted for publication December 23, 2020

Published online September 26, 2021

\begin{abstract}
The Ontario Cancer Profiles is an interactive dashboard for the public containing population-level cancer statistics created by Ontario Health (Cancer Care Ontario). The tool contains data on cancer burden, cancer screening measures, and cancer risk factors by Local Health Integration Network (LHIN) and Public Health Unit (PHU). It can be used for health system planning, measuring health systems performance, monitoring the impact of interventions, and to help identify new areas of research. There were 9 new modifiable cancer risk factors proposed to be included in future updates of the dashboard. The proposed risk factors include: access to care, active transportation, binge drinking, alcohol abstinence, inadequate fruit consumption, inadequate vegetable consumption, sedentary behaviour, second-hand smoke exposure, and sun safety. My practicum consisted of two main objectives: to conduct a literature review on the association between the proposed risk factors and cancer and to determine the prevalence of exposure of the identified risk factors in Ontario using 2015 to 2017 CCHS data. I performed a literature review to examine current evidence linking each proposed risk factor with cancer risk to determine the inclusion or exclusion of the indicator in the analysis. An analysis was performed with the selected variables in CCHS. Each indicator was age-standardized, and both standardized and crude ratios of individuals engaging in selected indicator activities were calculated. The results were examined for reliability using the produced coefficient of variation values. The estimates for each risk indicators allowed for the identification of target population that may be at higher risk of developing cancer due to greater exposure to the risk factors. They also serve as useful predictors for areas of improvement in regions with a high prevalence, such as healthy living within the community, and a guide to implementing preventative measures, screening, or treatment plans that may have been lacking.
\end{abstract}

